\title{
Estudio del confort térmico en las aulas de la Escuela Técnica Superior de Ingeniería de Edificación de la Universidad de Granada. Study of thermal comfort in the classrooms of the Technical School of Building Engineering of the University of Granada.
}

\author{
Alicia Anguita $^{\mathrm{a}}$, Julián Arco $^{\mathrm{b}}$, David Hidalgo $^{\mathrm{b}}$ \\ a Empresa Eipm. (aliciaanguita@correo.ugr.es). b Universidad de Granada. (Juliannn@ugr.es; dhidalgo@ugr.es)
}

Resumen - En las últimas décadas, se está produciendo un incremento de los estudios de las condiciones ambientales interiores de los edificios motivado, fundamentalmente, por el aumento del tiempo de permanencia de las personas en el interior de los mismos. La posibilidad de ajustar las condiciones de confort ambiental interior no solo mejora la calidad de vida de las personas, sino que puede constituir un elemento fundamental tanto en el control del gasto energético como en el control de las emisiones a la atmosfera. Los Estándares de confort térmico se dividen en cuantitativos, (Fanger e ISO 7730) y adaptativos, (ASHRAE 55 y UNE-EN 15251). Aunque los últimos estudios publicados discurren en la línea de considerar que los adaptativos ofrecen resultados más reales, los cuantitativos alcanzan resultados importantes si se complementan con encuestas de opinión de los usuarios. La presente investigación tiene como objetivo estudiar las condiciones de confort de dos aulas de la Escuela Técnica Superior de Ingeniería de Edificación de la Universidad de Granada, en base a lo establecido en la norma ISO 7730 y, a continuación, comparar los valores obtenidos con las opiniones de los usuarios recabadas mediante encuestas de sensación térmica. Los resultados alcanzados por ambos métodos difieren de manera considerable durante el intervalo de tiempo estudiado.

Palabras clave - Confort térmico; Edificación; Eficiencia energética; Habitabilidad.

\begin{abstract}
In the last decades, an increase of the studies of the interior environmental conditions of the buildings is taking place, motivated, fundamentally, by the increase of the time of permanence of the people inside them. The possibility of adjusting indoor environmental comfort conditions not only improves the quality of life of people but can also be a fundamental element in both the control of energy expenditure and the control of emissions to the atmosphere. The thermal comfort standards are divided into quantitative (Fanger and ISO 7730) and adaptive (ASHRAE 55 and UNE-EN 15251). Although the latest published studies run in the line of considering that adaptive offer more real results, quantitative results reach important results if they are complemented with user opinion surveys. The objective of this research is to study the comfort conditions of two classrooms of the School of Building Engineering of the University of Granada, based on what is established in the ISO 7730 standard, and then to compare the values obtained with the user opinions collected by thermal sensing surveys. The results achieved by both methods differ considerably during the time interval studied.
\end{abstract}

Index Terms - Thermal comfort; Edification; Energy efficiency; Habitability.

Alicia Anguita Aranda trabaja como técnico BIM en la empresa de ingeniera y Proyect Manager Eipm.
Julián Arco Díaz y David Hidalgo García son PhD en el Departamento de Expresión Gráfica Arquitectónica y en la Ingeniería. Escuela Técnica Superior de Ingeniería de Edificación de Granada. 


\section{INTRODUCCIÓN}

$\mathrm{D}$ esde los orígenes de la civilización, las personas han tenido que desarrollar una tendencia natural de adaptación a las condiciones climáticas de su entorno. Estas, no solo han determinado nuestra forma de vida sino también, la ubicación geográfica de las ciudades, el comercio y las zonas de conflicto. En esta línea, el autor Olgyay (1988) indica que el hombre siempre se ha esforzado por llegar al punto en el que adaptarse a su entorno le requiere solamente un mínimo de energía. Esta teoría junto al desarrollo de una pequeña capacidad tecnológica fue lo que propició la aparición de la agricultura y la construcción de las primeras grandes civilizaciones: Mesopotámica, Egipcia, Minoica, Micénica, etc. Todas ellas, ubicadas en climas considerados templados, anexas al mar y con abundancia de agua.

El estudio y control de las condiciones de habitabilidad de los espacios interiores tampoco es nuevo. Así, las viviendas de las ciudades islámicas medievales ubicadas en climas cálidos disponían de patio central o lateral con vegetación y ventanas con persianas o contraventanas al objeto de mejorar las condiciones de confort ambiental de las dependencias. Posteriormente, en el siglo XVI y tras vincular las grandes epidemias que asolaba a la población europea con las condiciones de insalubridad en las ciudades y viviendas propició que los tratadistas renacentistas comenzaran con el estudio de las condiciones de iluminación, ventilación y soleamiento en las viviendas (Hidalgo et al, 2016). Estos dieron origen, dos siglos más tarde, al nacimiento de las teorías higienistas que han derivado a día de hoy en lo que se define como confort térmico.

El término cuenta con múltiples definiciones, así la American Society of Heating, Refrigerating and Air-Conditioning Enginers, (ASHRAE) indica que es "la condición de la mente que expresa satisfacción con el ambiente térmico" (ASHRAE, 2017). La norma ISO 7730 (2006) la define como "aquella condición mental que expresa satisfacción con el ambiente térmico" y el autor Nikolopoulou (2004) como "la satisfacción psicofisiológica del humano con respecto a las condiciones climáticas del entorno". Estas definiciones coinciden en que se produce confort térmico cuando las personas sienten satisfacción con el medio ambiente que les rodea. La definición que realiza el Instituto Nacional de Seguridad e Higiene en el Trabajo va en la misma línea aunque es más explícita en relación a lo que se entiende por satisfacción: "Cuando las personas no experimentan sensación de calor ni frio" (Guzmán et al, 2014); es decir, cuando las condiciones de temperatura, humedad y movimientos del aire son favorables a la actividad que se desarrolla" (INSHT, 2007).

A diario las personas estamos sometidas a cambios de temperatura, humedad y velocidad del aire. Consideramos que se produce confort térmico ambiental cuando no existe ningún tipo de incomodidad térmica, por tanto, no sentimos ni frio ni calor. Esta sensación es definida por el autor Givoni, (1989: 7) como: "la ausencia de irritación o malestar térmico".

El sector de la construcción contribuye de manera importante en el deterioro del medio ambiente en sus distintas fases, (extracción y fabricación de materiales, instalación, gestión de la producción y residuos). Según los datos del Instituto para la Diversificación y Ahorro de Energía (IDEA), en el año 2016, el sector residencial español consumió el $16 \%$ de la energía total primaria por sectores. De esta, el $43 \%$ fue destinado a la instalación de calefacción y refrigeración. A su vez, los edificios generaron el $19 \%$ de las emisiones globales contaminantes, incluidas las producidas para su consumo en dicho sector (Lucon et al., 2014). Estos datos confrontan con la Estrategia Europea 2020 puesta en marcha por la Comisión en el año 2010 (Comisión Europea, 2008) y ampliada en valor y plazo en el año 2014 hasta el año 2030 (Sánchez et al, 2017).

Existen numerosos estudios que demuestran que una reducción en las temperaturas ambientales de calefacción y refrigeración disminuye considerablemente el consumo energético (Sánchez et al, 2017: 8). Así, los autores Spyropoulos y Balaras (2011) obtuvieron una reducción del $45 \%$ en el consumo energético ajustando la temperatura de calefacción a $20^{\circ} \mathrm{C}$ y la de refrigeración a $26^{\circ} \mathrm{C}$.

Por contra, el Reglamento de Instalaciones Térmicas en Edificios (RITE) vigente en España y de obligado cumplimiento establece unas condiciones de confort muy estrictas con respecto a la temperatura operativa y la humedad relativa. Con respecto al primer parámetro, el RITE establece unos valores de entre 21 y $23^{\circ} \mathrm{C}$ en invierno y de entre 23 y $25^{\circ} \mathrm{C}$ en verano. Con respecto a la humedad relativa los valores para el verano y el invierno son de entre el 30 y el $70 \%$. Estos estrictos rangos de temperatura dejan poco margen para estudiar una posible reducción en el consumo energético sin disminuir las condiciones de confort ambiental.

El presente artículo expone el estudio llevado a cabo en dos aulas de la Escuela Técnica Superior de Ingeniería de Edificación de la Universidad de Granada sobre su confort térmico.

Además, los métodos de evaluación del confort térmico presentan dos enfoques bien diferenciados: por un lado el Cuantitativo y por otro el Adaptativo. El primero se basa en la transferencia de calor entre el cuerpo de las personas y el ambiente. El precursor de este método fue Povl Fanger en el año 1970 que lo denominó modelo de balance térmico. Este va asociado a los indicadores PMV (Voto Medio Estimado) y PPD (Porcentaje Estimado de Insatisfechos). El primero representa el voto medio previsto en la escala de sensación térmica de un 
grupo de personas expuestas a un cierto ambiente. Este establece una tensión térmica basada en la transferencia de calor en estado estacionario entre el cuerpo y el ambiente y asigna un voto de confort a esa cantidad de tensión. Por tanto, el PPD es el porcentaje previsto de personas insatisfechas en cada PMV (Marincic, 2010). En la actualidad el método del balance térmico ha derivado en la norma ISO-7730. Los autores Sánchez García Et al definen este modelo como "los que toman al ocupante como un receptor de estímulos térmicos" (Sánchez et al, 2017: 9).

Por el contrario, los de enfoque adaptativo tienen en cuenta el proceso de adaptación de las personas cuando se produce la transferencia de calor con el ambiente. No solo a nivel físico, sino también a nivel psicológico y fisiológico. Por tanto, especifican las combinaciones de los factores humanos o personales y de las condiciones térmico-ambientales más adecuadas con el fin de suministrar y satisfacer a la mayoría de las personas que trabajan o residen en un edificio (Marincic, 2010). Dentro de los modelos de confort adaptativo destacan las normas ASHRAE 55 y las EN-15251.

Los modelos de evaluación han evolucionado con el paso del tiempo, así las aportaciones de los autores De Gear y Bragger (1997), (1998) y Givoni (1998) han permitido ajustar las condiciones de confort térmico dependiendo de la ubicación geográfica, climatología y preferencias térmicas de las personas.

En líneas generales, los estudios adaptativos son considerados como los que permiten establecer resultados más reales en relación al confort térmico efectivo de las personas, aunque los modelos cuantitativos alcanzan resultados importantes si se complementan con encuestas de opinión de los usuarios (Serra y Coch, 2005).

\section{OBJeto DE Estudio}

El objetivo principal de esta investigación consiste en la determinación de las condiciones de confort de dos aulas de la Escuela Técnica Superior de Ingeniería de Edificación de la Universidad de Granada, en base a lo establecido en la norma ISO 7730: 2006 y a continuación, comparar los resultados obtenidos con las opiniones de los usuarios recabadas mediante encuestas de sensación térmica.

\section{A. Ubicación}

La Escuela Técnica Superior de Ingeniería de Edificación se encuentra ubicada en el centro de la ciudad de Granada y administrativamente incluido dentro del Campus Centro, $\left(37^{\circ}\right.$ $\left.11^{\prime} 5^{\prime \prime} \mathrm{N}, 3^{\circ} 36^{\prime} 4^{\prime \prime} \mathrm{W}\right)$. La orientación es hacia los cuatro puntos cardinales con una diferencia hacia el Noreste de $23^{\circ}$. Se ubica en una parcela con una superficie de $14.400 \mathrm{~m}^{2}$, la superficie construida del edificio es de $11.115 \mathrm{~m}^{2}$ de los que $2.981 \mathrm{~m}^{2}$ tienen un uso de aulas. El edificio fue inaugurado en el año 1969, obra de los arquitectos Carlos Pfeifer y Alberto López.
$\mathrm{Su}$ desarrollo se realiza en torno a un gran patio central disponiendo de tres lados con tres plantas de altura y un lado con cinco plantas de altura. Cuenta con múltiples aulas docentes, servicios administrativos, despachos, aulas de prácticas, salas de estudio, aulas de informática, salón de actos con capacidad para trescientas personas y cafetería. El horario de funcionamiento de la Escuela es de 8:00 am hasta las 21:30 horas, dando un cómputo total de 2.522 horas anuales.

En la última década se han acometido varias intervenciones de mejora y reforma, destacando principalmente la sustitución de carpinterías en ventanas, colocación de toldos, redistribución de aulas y sustitución de la caldera de gasoil para la instalación de calefacción por otra de gas ciudad de mayor eficiencia. La instalación se encuentra domotizada desde el área de instalaciones de la Universidad ubicada en el complejo administrativo del Triunfo. Esta circunstancia genera frecuentemente quejas de los usuarios por situaciones de disconfort ambiental en aulas y despachos.

\section{B. Clima}

La ciudad de Granada se encuentra a una altitud de 680 metros sobre el nivel del mar y en la falda de Sierra Nevada. Esta circunstancia condiciona de forma considerable su clima. De acuerdo con la clasificación climática de koppen-geiger, cuenta con un clima de transición entre el clima mediterráneo (Csa) y el clima semiárido frio (Bsk) lo que implica inviernos húmedos y suaves y veranos cálidos y secos. Sierra Nevada es el macizo montañoso con mayor altitud de toda Europa Occidental, después de los Alpes. Su altitud máxima se alcanza en el pico del Mulhacén con 3.482 metros, aunque las altitudes medias de todo el macizo son de 2.400 metros, circunstancia que provoca una importante amplitud térmica anual.

La temperatura promedio anual fluctúa entre $\operatorname{los} 2,6^{\circ} \mathrm{C}$ y los $34,8^{\circ} \mathrm{C}$ con extremas en verano de $44^{\circ} \mathrm{C}$ y de $-4^{\circ} \mathrm{C}$ en invierno. El número medio de días al año con heladas es de 48. En cuanto al régimen térmico, el invierno es frio y se prolonga durante los meses de diciembre a febrero. El verano es la estación más larga, siendo Julio el mes en el que se alcanzan las temperaturas más altas (AEMET, 2019).

La precipitación media anual ronda valores de entre 350-400 mm., con una media de 52 días de lluvia al año concentrándose durante el Otoño y la Primavera. El promedio de la humedad relativa es de $58 \%$ con una mínima de $35 \%$ durante el verano. El número medio de horas de sol al año es de 2917 horas (AEMET, 2019), dando una media de 7,99 horas de sol al día.

\section{Sistemas constructivos e instalaciones}

El edificio, (figura 1), fue construido mediante una cimentación, estructura y pilares de hormigón armado. Los cerramientos mediante fábrica de medio pie de ladrillo a cara vista, enfoscado por el interior, cámara de aire sin aislamiento de 7 cm., tabicón de $7 \mathrm{~cm}$ y guarnecido interiormente. La 
transmitancia térmica del cerramiento es de $1,48 \mathrm{~W} / \mathrm{m}^{2} \mathrm{~K}$. Las ventanas cuentan con vidrios $6+8+6$, de $2,95 \mathrm{~W} / \mathrm{m}^{2} \mathrm{~K}$ y marcos de aluminio de $4,60 \mathrm{~W} / \mathrm{m}^{2} \mathrm{~K}$. Las cubiertas son no transitables con terminación mediante grava blanca. Por cuestiones estéticas la estructura sobresale de los cerramientos generando un volumen importante de puentes térmicos.

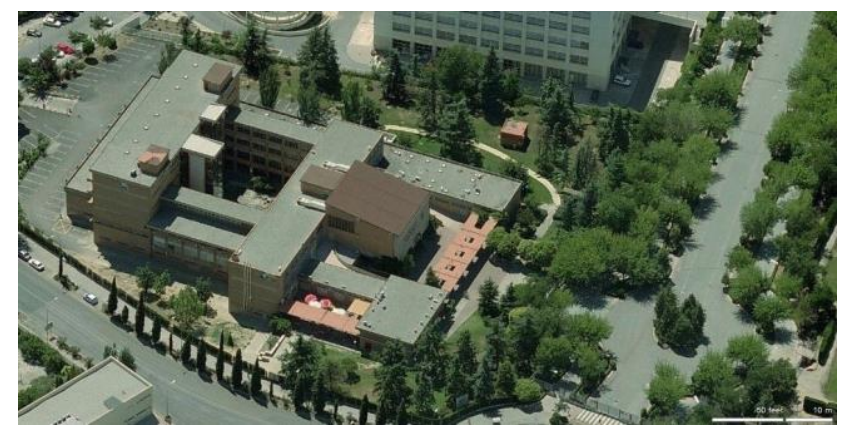

Fig. 1. Escuela Técnica Superior de Ingeniería de Edificación de la Universidad de Granada. Fuente: Bing.com.

La instalación de calefacción dispone de tres calderas de gas ciudad con el fin de conseguir que la producción centralizada de calor se aproxime lo más posible al régimen de rendimiento máximo. Dispone un fraccionamiento de la potencia en tres partes iguales, de forma que la suma de las potencias de los generadores de calor se ajusta a la suma de las demandas máximas simultáneas de la instalación, desarrollando éstas una potencia de $900 \mathrm{KW}$. El sistema se realiza mediante cinco circuitos independientes de forma que cada uno abastece a zonas de igual orientación y utilización. El sistema consiste en impulsión de agua caliente mediante bomba con sistema bitubular para la distribución del fluido calefactor. El número de elementos de radiador instalados en el edificio ronda los 4.900 elementos aproximadamente. Durante los meses de invierno el sistema de calefacción funciona por la mañana entre las 7:30 y las 12:30 horas y por la tarde, entre las 15:30 y las 19:30 horas, disponiendo de unas temperaturas de consigna de entre $21^{\circ} \mathrm{C}$ y $23^{\circ} \mathrm{C}$. El edificio no cuenta con instalación de refrigeración, de tal forma que, durante los meses de mayor temperatura, la ventilación se realiza de forma natural mediante ventanas que abren y cierran los propios usuarios.

\section{MetodologíA}

Al objeto de cumplir con el objetivo anteriormente indicado, se ha llevado a cabo la metodología establecida en la figura 2. Los apartados pormenorizados son los que a continuación se detallan.

\section{A. Elementos de monitorización}

Los elementos de monitorización de las aulas utilizados en la investigación han sido sondas data logger de temperatura, termohigrómetro para la humedad relativa y termoanemómetro de molinete para la velocidad del aire; todos de la marca Testo.

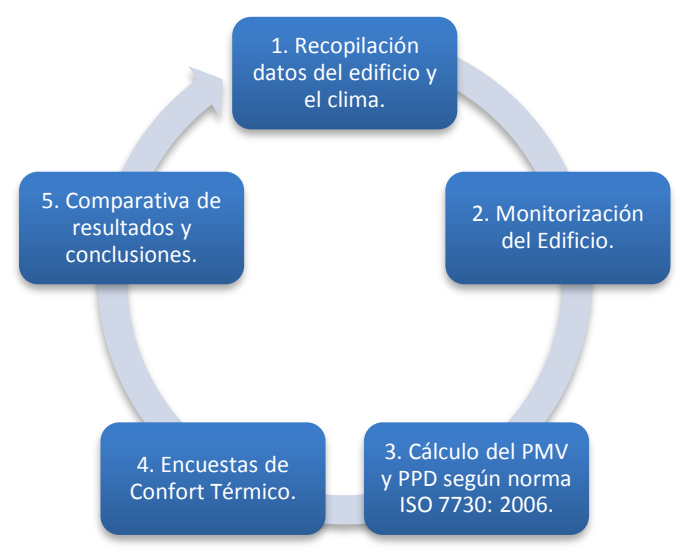

Fig. 2. Proceso metodológico llevado a cabo en la investigación.

El modelo de data logger utilizado para medir y registrar la temperatura ha sido el 174T (figura 3). Registra los valores de temperatura de manera segura garantizando así una documentación y registro ininterrumpido de los datos de medición.
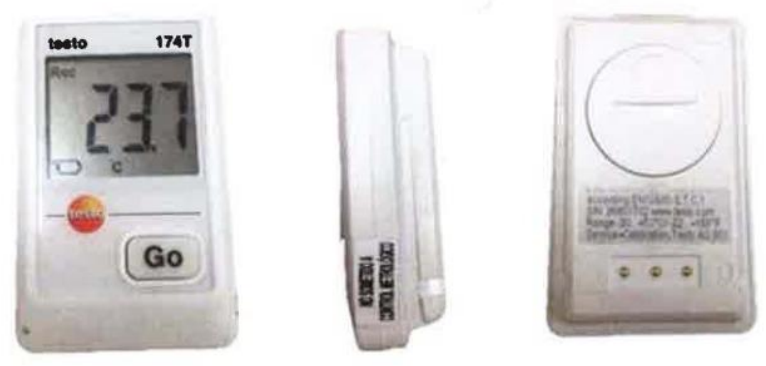

Fig. 3. Data logger de temperatura modelo $174 \mathrm{~T}$ de la marca Testo utilizado para la monitorización de las temperaturas.

El modelo de termohigrómetro utilizado para medir la humedad relativa de las aulas ha sido el 625. Permite la medición de la temperatura pero no registra los datos. Por tanto, las mediciones de humedad registradas han sido en los momentos en los que se han realizado las encuestas de confort ambiental.

El modelo de termoanemómetro utilizado para medir la velocidad relativa del aire ha sido el 417 que cuenta con molinete de $100 \mathrm{~mm}$. Este tampoco registra los datos por lo que las mediciones de velocidad del aire han sido realizadas en los momentos en los que se han realizado las encuestas de confort térmico.

\section{B. Monitorización del edificio}

Las aulas seleccionadas para la investigación han sido las denominadas D-12B, ubicada en la planta baja y la D21, ubicada en la planta primera. Ambas cuentan con una superficie aproximada de 100 metros, capacidad para 50 personas y están 
destinadas a clases prácticas por lo que presentan una mayor ergonomía y comodidad. La fachada exterior de la primera se encuentra orientada al Oeste, por tanto, recibe irradiación solar en horario de tarde mientras que las fachadas exteriores de la segunda se encuentran orientadas al Este, Noreste y Suroeste, por lo que recibe irradiación solar en horario de mañana.

Se han tomado medidas durante los meses de Febrero hasta Junio de 2018, de tal forma que se han abarcado las estaciones de invierno y primavera. Durante el verano, la escuela se encuentra cerrada o sin uso docente y el clima de la temporada de otoño es templado.

En ambas aulas se han colocado tres sondas de temperatura, (figura 4): exterior, interior a 0,80 metros de altura e interior a 2,40 metros de altura. Se programaron para que todas las sondas registraran la temperatura cada 15 minutos, obteniendo 14.400 registros por sonda y un total de 86.400 registros.

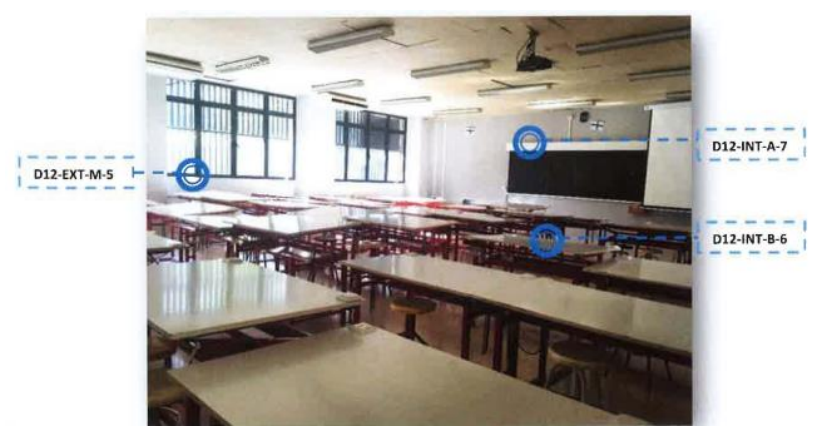

Fig. 4. Aula D12 con la ubicación de las sondas de temperatura.

Las exteriores fueron ubicadas sobre el alfeizar de una ventana y dentro de un tarro esférico de PVC de color claro para que no verse afectadas por las radiaciones solares. El envoltorio cuenta con taladros calibrados en todo su perímetro al objeto de atenuar al máximo las alteraciones de temperatura. También se cerraron las lamas verticales de protección solar de esa ventana al objeto de eliminar la radiación directa sobre el tarro.

Las sondas interiores inferiores se colocaron colgadas de una de las patas de una mesa ubicada en la fila central y a 0,80 metros del suelo (figura 5). Las sondas interiores superiores se colocaron encima de la pizarra a una altura de 2,40 metros del suelo.

Los datos de temperatura registrados por las sondas fueron trasladados a tablas de Excel y posteriormente, a gráficas con la

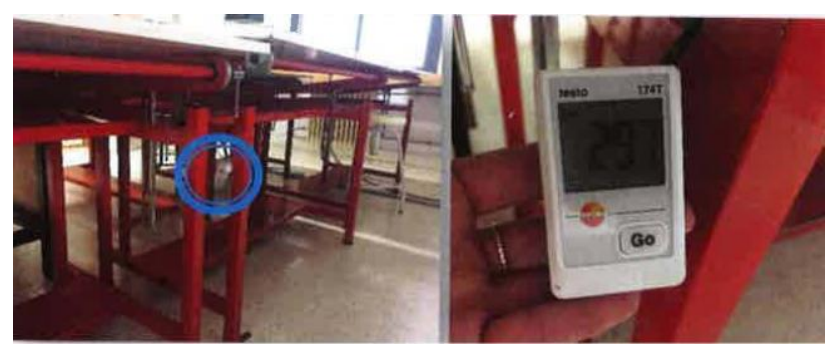

Fig. 5. Sonda temperatura de altura intermedia colocada en aula. determinación de los rangos de confort establecidos en el RITE y el momento en el que se desarrolló la encuesta de confort ambiental, (figura 6).

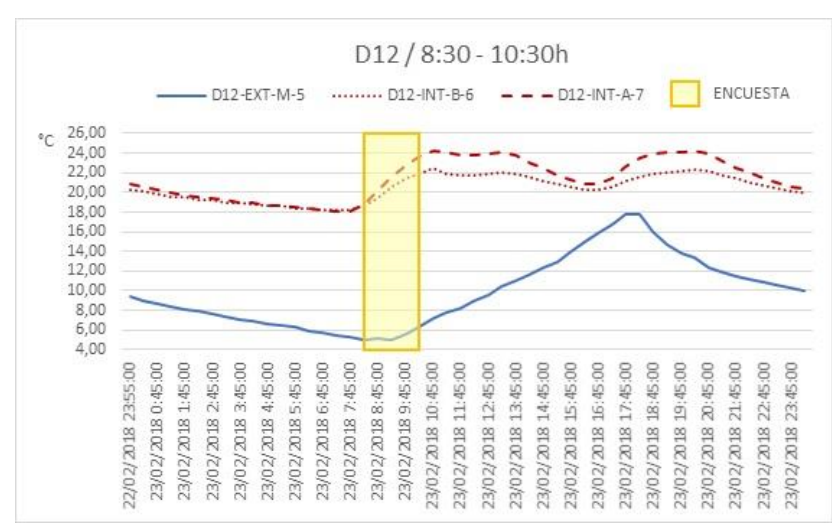

Fig. 6. Gráfica de temperaturas registradas y horario de realización de encuesta de confort ambiental.

\section{Cálculo del PMV y PPD según norma ISO 7730: 2006.}

La sensación térmica de las personas está relacionada, principalmente, con el equilibrio global de su cuerpo. Este depende de la actividad física y de la vestimenta, así como de la temperatura del aire, temperatura radiante media, velocidad y humedad del aire (ISO 7730:2006)

El Voto Medio Estimado (PMV) indica el valor medio de los votos emitidos por un grupo de personas respecto de una escala térmica de 7 niveles, (figura 7), basado en el equilibrio térmico del cuerpo humano. El equilibrio térmico se consigue cuando la producción de calor interna es igual a su perdida hacia el ambiente. La norma ISO 7730:2006 establece que los valores térmicos recomendados deben proporcionar bienestar térmico global al $90 \%$ de los usuarios. Esta circunstancia implica considerar como personas insatisfechas las que votaron las opciones: muy caluroso (+3), caluroso $(+2)$, fresco $(-2)$ o frio $(-$ 3).

\begin{tabular}{|c|c|l|}
\hline \multicolumn{2}{|c|}{ Puntuación } & Sensación térmica \\
\hline+3 & Sofocante \\
\hline+2 & Calor \\
\hline+1 & Ligeramente cálido \\
\hline 0 & Neutral \\
\hline-1 & Ligeramente frío \\
\hline-2 & Fresco \\
\hline-3 & Frío \\
\hline
\end{tabular}

Fig. 7. Escala valores térmicos según Norma ISO 7730.

El Porcentaje Estimado de Insatisfechos (PPD) establece una predicción cuantitativa del porcentaje de personas que no sentirán satisfacción ambiental por notar frio o calor. Este se calcula cuantitativamente mediante la fórmula 10 aproximadamente mediante la gráfica de la figura 8 . 


$$
P P D=100-95 \mathrm{oexp}\left(-0,03353 \mathrm{o} P M V^{4}-0,2179 \mathrm{o} P M V^{2}\right)
$$

Para proceder al cálculo del PMV, se han tenido en cuenta los siguientes valores establecidos en la ISO 7730:2006:

- Tasa metabólica por actividad. Según Anexo B, tabla B.1, la tasa metabólica elegida es de $58 \mathrm{~W} / \mathrm{m} 2$ o 1.0 met, cuya actividad se corresponde con la situación de reposo, sentado.

- Aislamiento térmico para combinación de ropa habitual. En las visitas realizadas a las aulas se ha tenido en cuenta la ropa con la que el alumnado venía a clase. Esta se ha asimilado a la que aparece en el Anexo C, tabla C.1 de la norma. De esta forma, el aislamiento térmico para combinación de ropa elegida ha sido de 1.00 Clo o 0.155 $\mathrm{m}^{2} \mathrm{~K} / \mathrm{W}$ para la temporada de invierno y 0.5 Clo o 0.080 $\mathrm{m}^{2} \mathrm{~K} / \mathrm{W}$ para la temporada de primavera.

- Aislamiento térmico para asientos. Las sillas son metálicas con asientos de madera. Según la tabla C.3 de la norma, el valor elegido ha sido de 0.00 clo y $0.00 \mathrm{~m}^{2} \mathrm{~K} / \mathrm{W}$.

- Temperatura radiante media. Tal y como se ha indicado, se han colocado dos sondas interiores en cada aula, una a 0,80 metros del suelo y otra a 2,40 metros. Para obtener la temperatura radiante media se ha tenido en cuenta la temperatura de la sonda superior en una proporción de 1/3 y la temperatura de la sonda inferior en $2 / 3$.

El cálculo del PMV y PPD se ha realizado mediante la aplicación informática de evaluación del confort térmico con el método de Fanger de Ergonautas (Diego, 2015).

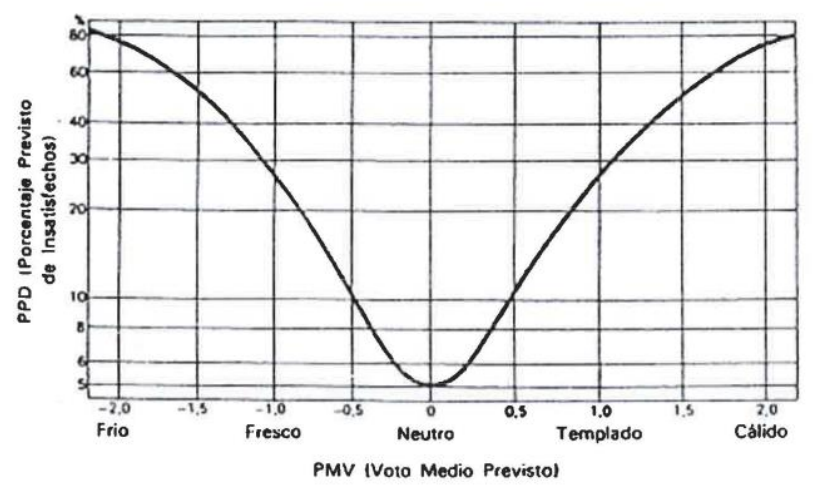

Fig. 8. Cálculo de PPD en función de PMV. Fuente: Norma ISO 7730:2006, pp. 11.

\section{Encuestas de Confort Térmico}

A través de las encuestas es posible conocer y analizar las sensaciones térmicas de los usuarios en las aulas. Este proceso se llevó a cabo mediante la participación directa de los mismos mediante un cuestionario realizado in situ por los investigadores. El cuestionario consta de cuatro apartados: datos generales de los encuestados: edad, sexo, nacionalidad y lugar de residencia; datos de observación: peso, estatura y vestimenta; sensación térmica experimentada en el momento de la evaluación: según escala de sensación térmica de siete puntos indicado en la norma ISO 7730:2006 y por último, datos de comportamiento previo: actividad previa e ingesta de comida, (figura 9).

Con los datos del apartado de observación se obtuvo el Índice de Masa Corporal (IMC) de cada participante según el método establecido por la Organización Mundial de la Salud (OMS).

Las encuestas fueron realizadas en las dos aulas los días 23 de febrero y 2 de Marzo a las 9:30 horas durante la temporada de invierno, y los días 6 y 27 de Abril, 28 y 30 de Mayo a las 11:30 horas durante la temporada de primavera.

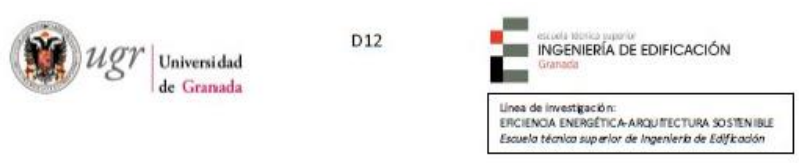

ENCUESTA CONFORT TÉRMICO ADAPTATIVO

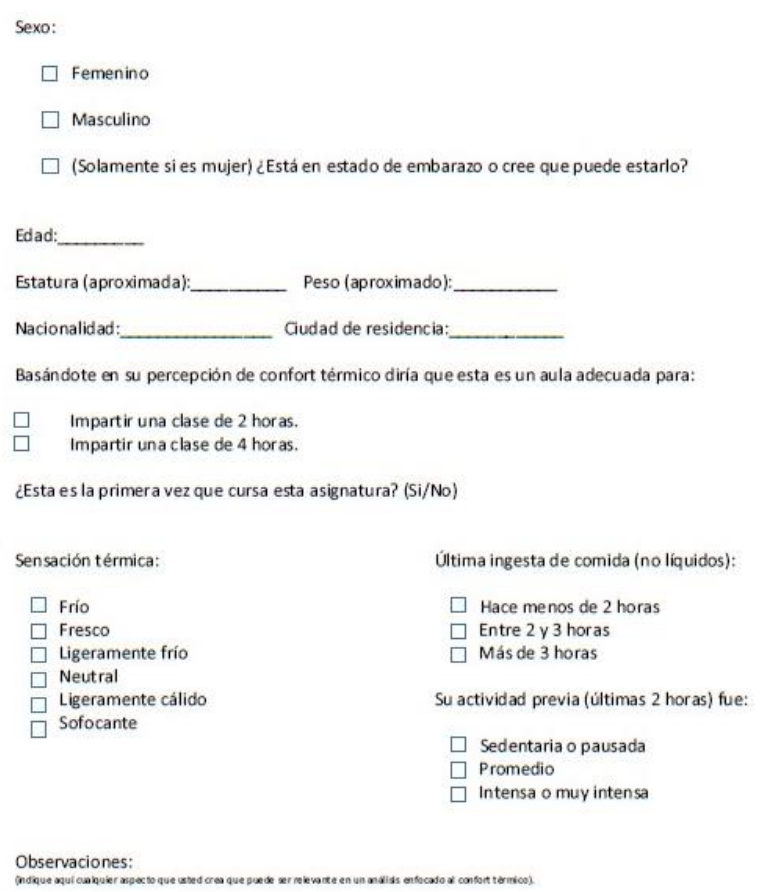

Fig. 9. Modelo de encuesta de satisfacción ambiental.

\section{RESUltadOS Y DiscUSIÓN}

Los resultados de las mediciones y de las encuestas de confort ambiental, son los siguientes:

\section{A. Mediciones temporada de invierno}

Los datos de la tabla I reflejan los valores medios obtenidos por las sondas de temperatura durante el tiempo que se tardó en realizar la encuesta entre los usuarios del aula. Los días indicados en la tabla se obtuvieron unas mediciones de velocidad del aire de $0,5 \mathrm{~m} / \mathrm{s}$ y una humedad relativa del $30 \%$. En base a estos parámetros y mediante la aplicación informática de evaluación del confort térmico con el método de Fanger de 
Ergonautas (Diego, 2015), se obtuvieron los siguientes datos de PMV y PPD.

TABLA I

DATOS SONDAS TEMPERATURA

\begin{tabular}{ccccc}
\hline \hline Día & Aula & $\begin{array}{c}\text { T. M. } \\
\text { Exterior } \\
\left({ }^{\circ} \mathrm{C}\right)\end{array}$ & $\begin{array}{c}\text { T.M. } \\
\text { Interior } \\
\text { Inferior } \\
\left({ }^{\circ} \mathrm{C}\right)\end{array}$ & $\begin{array}{c}\text { T.M. Interior } \\
\text { Superior }\left({ }^{\circ} \mathrm{C}\right)\end{array}$ \\
\hline $23 / 02 / 18$ & D-12 & 5,75 & 21,81 & 20,73 \\
$02 / 03 / 18$ & D-12 & 13,4 & 21,83 & 20,75 \\
$23 / 02 / 18$ & D-21 & 9,31 & 22,40 & 20,30 \\
$02 / 03 / 18$ & D-21 & 12,31 & 20,60 & 19,56 \\
\hline \hline
\end{tabular}

Los datos obtenidos, (tabla II) presentan valores similares en ambas aulas estableciendo un porcentaje de personas con disconfort ambiental de entre el 15,01 y el 23,66\%.

\section{B. Sensación térmica en temporada de invierno}

La tabla III refleja los resultados de confort obtenidos mediante encuestas de satisfacción de los usuarios en la temporada de invierno. La ocupación media de las aulas fue constante, rondando los 30-38 usuarios. Resaltar que ninguno de ellos marcó las situaciones ambientales denominadas "cálido" y "muy cálido". Respecto a la sensación térmica percibida por los usuarios del aula D12, el 2\% respondió encontrarse en una situación de frio, el 15\% ligeramente frio, el $63 \%$ situación neutral y el 19,56\% situación de ligeramente cálido. Con respecto a los usuarios del aula D21, el 5\% respondió encontrarse en una situación de frio, el 6,9\% fresco, el $22,22 \%$ ligeramente frio, el $50 \%$ situación neutral y el $16,66 \%$ ligeramente cálido. El total de encuestas obtenidas para ambas aulas fue de 119 y el mayor porcentaje de respuestas correspondió a la opción de neutro.

TABLA II

CÁLCULO DE PMV Y PPD SEGÚN APLICACIÓN

\begin{tabular}{ccccc}
\hline \hline Día & Aula & $\begin{array}{c}\text { T. M. } \\
\text { Radiante }\end{array}$ & PMV & PPD (\%) \\
\hline $23 / 02 / 18$ & D-12 & 20,98 & 0,69 & 15,01 \\
$02 / 03 / 18$ & D-12 & 21,11 & 0,69 & 15,01 \\
$23 / 02 / 18$ & D-21 & 21,00 & 0,69 & 15,01 \\
$02 / 03 / 18$ & D-21 & 19,90 & 0,94 & 23,66 \\
& & & & \\
\hline \hline
\end{tabular}

En relación con el Índice de Masa Corporal de los usuarios, indicar que 32 de los encuestados presentaban sobrepeso y el $46 \%$ de estos seleccionaron alguna opción de disconfort ambiental. En concreto, el 93\% marcó la opción ligeramente cálida y el $7 \%$ ligeramente frio. 3 usuarios presentaban

TABLA III

RESULTADOS CUESTIONARIO SENSACIÓN TÉRMICA

\begin{tabular}{ccccccc}
\multicolumn{7}{c}{ RESULTADOS CUESTIONARIO SENSACION TERMICA } \\
\hline \hline Día & Aula & Frio & $\begin{array}{c}\text { Fresc } \\
\mathrm{o}\end{array}$ & $\begin{array}{c}\text { Ligera } \\
\text { mente } \\
\text { frio }\end{array}$ & Neutro & $\begin{array}{c}\text { Ligera } \\
\text { mente } \\
\text { cálido }\end{array}$ \\
\hline $23 / 02 / 18$ & D-12 & 1 & 0 & 3 & 17 & 5 \\
$02 / 03 / 18$ & D-12 & 0 & 0 & 4 & 12 & 4 \\
$23 / 02 / 18$ & D-21 & 1 & 1 & 6 & 18 & 9 \\
$02 / 03 / 18$ & D-21 & 3 & 4 & 10 & 18 & 3 \\
& & & & & & \\
\hline \hline
\end{tabular}

infrapeso y el 33\% seleccionó alguna opción de disconfort ambiental. En concreto, el 100\% marcó ligeramente frio.

\section{Mediciones temporada de primavera}

Los datos de la tabla IV reflejan los valores medios obtenidos por las sondas de temperatura durante el tiempo que se tardó en realizar la encuesta entre los usuarios del aula Los días indicados en la tabla se obtuvieron unas mediciones de velocidad del aire de $0,5 \mathrm{~m} / \mathrm{s}$ y una humedad relativa del $30 \%$. En base a estos parámetros y mediante la aplicación informática de evaluación del confort térmico con el método de Fanger de Ergonautas (Diego, 2015), se obtuvieron los siguientes datos de PMV y PPD.

TABLA IV

DATOS SONDAS TEMPERATURA

\begin{tabular}{ccccc}
\hline \hline Día & Aula & $\begin{array}{c}\text { T. M. } \\
\text { Exterior } \\
\left({ }^{\circ} \mathrm{C}\right)\end{array}$ & $\begin{array}{c}\text { T.M. } \\
\text { Interior } \\
\text { Inferior } \\
\left({ }^{\circ} \mathrm{C}\right)\end{array}$ & $\begin{array}{c}\text { T.M. Interior } \\
\text { Superior }\left({ }^{\circ} \mathrm{C}\right)\end{array}$ \\
\hline $06 / 04 / 18$ & D-12 & 17,05 & 25,08 & 23,30 \\
$27 / 04 / 18$ & D-12 & 18,06 & 23,05 & 21,98 \\
$28 / 05 / 18$ & D-12 & 15,66 & 21,88 & 21,53 \\
$30 / 05 / 18$ & D-12 & 16,16 & 21,60 & 21,38 \\
$06 / 04 / 18$ & D-21 & 16,28 & 22,60 & 21,55 \\
$27 / 04 / 18$ & D-21 & 17,80 & 23,71 & 22,65 \\
$28 / 05 / 18$ & D-21 & 15,30 & 24,80 & 23,70 \\
$30 / 05 / 18$ & D-21 & 15,80 & 25,05 & 23,90 \\
& & & & \\
\hline \hline
\end{tabular}

En esta ocasión, los datos obtenidos, (tabla V), no presentan valores similares en ambas aulas estableciendo un porcentaje de personas con disconfort ambiental de entre el 36 y $88 \%$ para el aula D-12 y de entre el 43 u el $71 \%$.

\section{Sensación térmica en temporada de primavera}

La tabla VI refleja los resultados de confort obtenidos mediante encuestas de satisfacción de los usuarios en la temporada de primavera. La ocupación media de las aulas fue constante, rondando los 30-38 usuarios. Resaltar que ninguno de ellos marcó las situaciones ambientales denominadas "cálido" y "muy cálido". Respecto a la sensación térmica percibida por los usuarios del aula D12, el 5,4\% respondió

TABLA V

CÁLCULO DE PMV Y PPD SEGÚN APLICACIÓN

\begin{tabular}{ccccc}
\hline \hline Día & Aula & $\begin{array}{c}\text { T. M. } \\
\text { Radiante }\end{array}$ & PMV & PPD (\%) \\
\hline $06 / 04 / 18$ & D-12 & 23,89 & 1,21 & 36 \\
& & & & \\
$27 / 04 / 18$ & D-12 & 22,33 & 1,58 & 55 \\
$28 / 05 / 18$ & D-12 & 21,64 & 1,83 & 68 \\
$30 / 05 / 18$ & D-12 & 21,45 & 2,30 & 63 \\
$06 / 04 / 18$ & D-21 & 22,68 & 1,72 & 43 \\
$27 / 04 / 18$ & D-21 & 23,57 & 1,35 & 71 \\
$28 / 05 / 18$ & D-21 & 24,43 & 1,89 & 71 \\
$30 / 05 / 18$ & D-21 & 24,66 & 1,89 & \\
\hline \hline
\end{tabular}

encontrarse en una situación de fresco, el 10,95\% en situación de ligeramente frio, el 54,79\% situación neutral y el 28,76\% situación de ligeramente cálido. Con respecto a los usuarios del 
aula D21, el 0,8\% respondió encontrarse en una situación de frio, el 1,6\% fresco, el 4,4\% ligeramente frio, el $68.14 \%$ situación neutral y el 24,77\% ligeramente cálido. El total de encuestas obtenidas para ambas aulas fue de 186 y el mayor porcentaje de respuestas correspondió a la opción de neutro.

TABLA VI

RESULTADOS CUESTIONARIO SENSACIÓN TÉRMICA

\begin{tabular}{ccccccc}
\hline \hline Día & Aula & Frio & $\begin{array}{c}\text { Fresc } \\
\text { o }\end{array}$ & $\begin{array}{c}\text { Ligera } \\
\text { mente } \\
\text { frio }\end{array}$ & Neutro & $\begin{array}{c}\text { Ligera } \\
\text { mente } \\
\text { cálido }\end{array}$ \\
\hline $06 / 04 / 18$ & D-12 & 0 & 0 & 1 & 5 & 8 \\
$27 / 04 / 18$ & D-12 & 0 & 1 & 0 & 9 & 5 \\
$28 / 05 / 18$ & D-12 & 0 & 0 & 2 & 6 & 2 \\
$30 / 05 / 18$ & D-12 & 0 & 3 & 5 & 20 & 6 \\
$06 / 04 / 18$ & D-21 & 1 & 0 & 3 & 17 & 5 \\
$27 / 04 / 18$ & D-21 & 0 & 2 & 1 & 18 & 9 \\
$28 / 05 / 18$ & D-21 & 0 & 0 & 1 & 18 & 9 \\
$30 / 05 / 18$ & D-21 & 0 & 0 & 0 & 22 & 8 \\
\hline \hline
\end{tabular}

En relación con el Índice de Masa Corporal de los usuarios, indicar que 44 de los encuestados presentaban sobrepeso y el $46 \%$ de estos seleccionaron alguna opción de disconfort ambiental. En concreto, el 85\% marcó la opción ligeramente cálida y el $15 \%$ ligeramente frio. 3 usuarios presentaban infrapeso y el 66\% seleccionó alguna opción de disconfort ambiental. En concreto, el 100\% marcó ligeramente frio.

\section{E. Discusión de los resultados}

Los resultados obtenidos para cada aula investigada según los métodos llevados a cabo, (ISO 7730 y encuestas de satisfacción de confort ambiental), se encuentran reflejados en la figura 10 .

Aplicando el método de determinación de PMV y PPD establecido en la norma ISO 7730 para las aulas D12 y D21 se obtuvieron unos porcentajes medios de disconfort ambiental en la temporada de invierno del $15,01 \%$ y $19,33 \%$ respectivamente. Sin embargo, los resultados obtenidos mediante las encuestas de satisfacción ambiental elevan esos resultados al doble de los calculados por la norma ISO. En concreto, los valores obtenidos han sido de $37,31 \%$ y $50,60 \%$ respectivamente.

En la temporada de primavera se produce una circunstancia inversa a la ocurrida en la temporada de invierno. Si el método de cálculo según la norma ISO 7730 otorga unos porcentajes de insatisfacción excesivamente elevados, $61,75 \%$ y $62 \%$ respectivamente, las encuestas de satisfacción reducen los valores obtenidos a casi la mitad, en concreto a $46,36 \%$ y $32 \%$, respectivamente.

Se observa que los porcentajes de disconfort ambiental establecidos por la norma ISO 7730 son similares en ambas aulas tanto en la temporada de invierno como en la de verano. Esta circunstancia se encuentra motivada en que las condiciones ambientales interiores de las aulas, (temperatura, humedad y velocidad del aire), han sido iguales y por tanto, el método otorga satisfacciones similares. Sin embargo, los datos de satisfacción de los usuarios de las aulas obtenidas mediante encuestas de satisfacción han reflejado resultados distintos.

Si se comparan las dos aulas objeto de estudio se observa que el factor orientación y el sistema constructivo del edificio influyen de manera considerable. Así, el aula D21 ubicada al Este y con tres fachadas sin aislamiento presenta un mayor porcentaje de insatisfacción en invierno que el aula D12 ubicada al Oeste y con una fachada exterior sin aislamiento. A mayor superficie exterior sin aislamiento, mayor disconfort ambiental. Por el contrario, en la temporada de primavera el aula D-12 con orientación oeste presenta un mayor porcentaje de disconfort ambiental que la D21, motivado por el calor que almacena el aula tras toda la tarde de radiación solar.

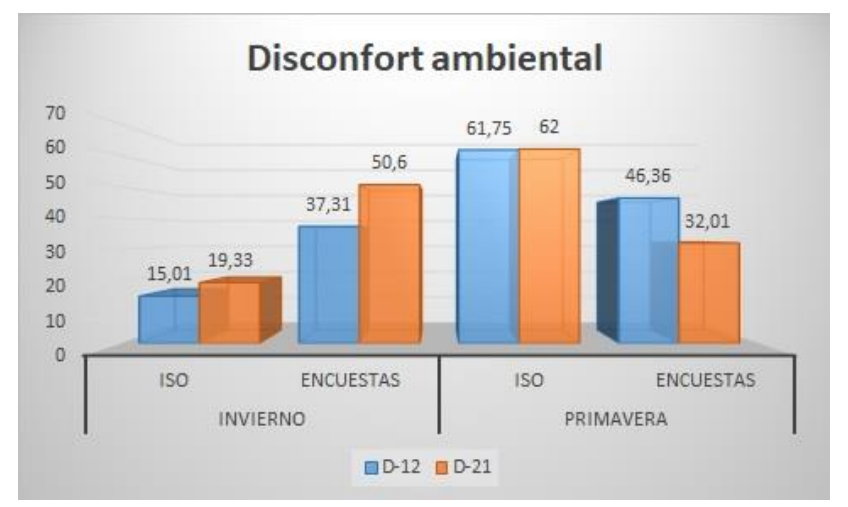

Fig. 10. Resultados en tanto por ciento de insatisfacción ambiental por aula y según modelo seguido.

La ventilación natural es otro factor que influye de forma considerable en la insatisfacción ambiental de la temporada de primavera. Si durante el invierno, las ventanas permanecen cerradas para evitar la entrada de aire frio, durante la primavera se convierten en un aliado ambiental máxime cuando el edificio no dispone de sistema de refrigeración. El aula D-12 dispone de tres ventanas alineadas, sin embargo, el aula D-21 cuenta con seis ventanas, enfrentadas tres a tres. Una mayor ventilación en la temporada de primavera cuando las temperaturas interiores suelen ser superiores a las exteriores mejora en el grado de satisfacción de los usuarios.

La ocupación media de ambas aulas durante el desarrollo de las encuestas de confort ambiental se ha mantenido constante, obteniendo una ocupación media de entre 30-38 personas.

\section{CONCLUSIONES}

El estudio de las condiciones de confort ambiental en el interior de los edificios viene desarrollándose desde hace algunas décadas y ha ido evolucionando a lo largo del tiempo. El progresivo desarrollo de este tipo de estudios e investigaciones se encuentra justificado por el aumento del tiempo de permanencia de las personas en el interior de los edificios que rondan valores de entre el $75-90 \%$ de nuestro 
tiempo (Piedecausa 2013). La posibilidad de ajustar las condiciones de confort ambiental al máximo no solo mejora la calidad de vida de las personas, sino que puede constituir un pilar fundamental tanto en el control del gasto energético como en el control de las emisiones a la atmosfera, disminuyendo, por tanto, el efecto conocido como cambio climático.

La sensación de confort ambiental de las personas se ve influenciada no solo por elementos ambientales, sino también por elementos fisiológicos y psicológicos. El estudio mediante la monitorización de todos ellos permite conocer con mayor detalle la satisfacción ambiental de los usuarios y por tanto, mejorar la calidad de vida de las personas.

En la investigación realizada en dos aulas de la Escuela Técnica Superior de Ingeniería de Edificación de la Universidad de Granada se han comparado los datos de satisfacción ambiental de los usuarios según la norma ISO 7730 y mediante encuestas de satisfacción. Para ello, se han monitorizado por un lado, los elementos ambientales mediante data logger de temperatura, termohigrómetro y termoanemómetro y por otro, los elementos fisiológicos $\mathrm{y}$ psicológicos mediante encuestas de satisfacción.

Los resultados obtenidos por ambos métodos difieren de manera considerable durante el intervalo de tiempo estudiado. Mediante el proceso de cálculo de PMV y PPD establecido en la norma ISO 7730 se obtienen resultados similares en ambas temporadas ya que las condiciones ambientales de las aulas han variado de forma análoga durante el tiempo objeto de estudio. Sin embargo, los datos resultantes de las encuestas de satisfacción difieren considerablemente de los anteriores. Así, el porcentaje de insatisfechos en la temporada de invierno se multiplica por dos mientras que en la temporada de primavera disminuye a la mitad. El motivo fundamental de esta disparidad de resultados radica en que las condiciones de confort ambiental influyen mediante una variedad de factores, algunos ya numerados anteriormente, tales como los psicológicos y los fisiológicos y otros no contemplados, tales como la orientación, sistemas constructivos, número y orientación de las ventanas y ocupación de los espacios. Estos también deben ser considerados si se pretende conocer realmente los valores de confort ambiental reales de los espacios interiores. No obstante, los autores Sánchez et al consideran que "el modelo PMV estima la sensación térmica de forma más precisa en edificios acondicionados mecánicamente que en edificios que cuentan con ventilación natural. Estas diferencias en la estimación pueden ser explicadas en gran medida por la menor dependencia de los edificios mecánicamente acondicionados de las temperaturas exteriores, y las menores posibilidades de adaptación, comparados con los edificios ventilados de forma natural" (2016: 39). En las dependencias objeto de nuestro estudio, y debido a la no existencia de sistemas de refrigeración, se ha necesitado complementar el método PMV mediante la realización de encuestas al objeto de poder conocer con mayor fiabilidad la satisfacción ambiental.

El conocimiento real de las condiciones de confort ambiental favorece el desarrollo de una planificación de espacios más habitables desde el momento del diseño y durante toda la vida útil de las edificaciones.

Por otro lado, y con respecto a las edificaciones ya ejecutadas, permite establecer o priorizar posibles intervenciones de mejora y acondicionamiento energético sobre todo en periodos de escasez de los recursos económicos. Así, para mejorar las condiciones de confort ambiental de las aulas objeto de estudio sería prioritario en la D-21 la colocación de un aislamiento en fachada que mejore las condiciones ambientales en la temporada de invierno. Por el contrario, en el aula D-12 cuyo disconfort ambiental es mayor en primavera sería prioritario la colocación de protectores solares en las ventanas, (tipo toldos, persianas de lamas abatibles o voladizos).

El enfoque cuantitativo sin modificaciones o alteraciones como sistema de conocimiento de las condiciones ambientales en el interior de los edificios no parece ser un método certero ya que como se ha comprobado, los datos obtenidos difieren con los indicados por los usuarios. Por tanto, una revisión de estos que permita incluir otros factores o una apuesta por los métodos adaptativos parece más adecuado que el simple enfoque cuantitativo.

\section{REFERENCIAS}

AENOR (2007). Ergonomía del ambiente térmico. Determinación analítica e interpretación del bienestar térmico mediante el cálculo de los índices PMV y PPD y los criterios de bienestar térmico local. UNE-EN ISO 7730:2007. Madrid: AENOR.

American Society of Heating Refrigerating and Air Conditioning Engineers (ASHRAE). (2017). Thermal environmental conditions for human occupancy. ASHRAE Standar 55-2017.

Agencia Española de Meteorología (AEMET). (2019). Datos meteorológicos de ciudades españolas. Consultado en: http://www.aemet.es/es/serviciosclimaticos/datosclimat ologicos/valoresclimatologicos? $1=5530 \mathrm{E} \& \mathrm{k}=$ and.

[Fecha de consulta: 10/01/2019].

Comisión Europea. (2008). Europe's climate change opportunity [en línea] Bruselas. Disponible en: http://eur-lex.europa.eu/legal-

content/EN/TXT/PDF/?uri=CELEX:52008DC0030\&fr om=EN. [Fecha de consulta:13/01/2019]

De Gear, R. (1997). Developing an adaptative model of thermal comfort and preference. Final Report RP-884. Sidney. 
De Gear, R. \& Braver, G. (1998). Thermal adaptation in the buil environment: a literature review. Energy and Building, (27), 83-96.

Diego-Mas, J. A. (2015). Evaluación del confort térmico con el método de Fanger. Ergonautas, Universidad Politécnica de Valencia. Disponible online: http://www.ergonautas.upv.es/metodos/fanger/fangerayuda.php. [Fecha consulta 13-01-2019].

Fanger, P.O. (1970). Thermal Comfort. Copenhagen. Danish: Technical Press.

Givoni, B. (1998). Climate considerations in building and urban design. John Wiley ans Sons, inc.

Guzmán, M.H. \& Ochoa, J.M. (2014). Confort térmico en los espacios públicos urbanos. Clima cálido y frio semiseco. Revista Hábitat sustentable, 4(2), 52-63.

Hidalgo, D. \& Santiago, J.M. (2016) Historia de la morfología urbana. Granada: AVIACAN.

Instituto Nacional de Seguridad e Higiene en el Trabajo (INSHT). (2007). Notas prácticas sobre confort térmico. Madrid: Instituto nacional de seguridad e higiene en el trabajo.

Lucon, O. \& Urge, D. \& Zain, A. (2014). Climate change 2014: Mitigation of climate change. Contribution of working group III to the first assesmente report of the intergovernmental panel on climate change. Bruselas: Comisión Europea, pp. 1-66.

Marincic, I. (2010). La vivienda económica en Hermosillo y el consume de energía eléctrica. En: XXXIV Semana Nacional de Energía Solar ANES 2010.

Ministerio de Industria, Energía y Turismo (2007). Reglamento de Instalaciones Térmicas en los Edificios (RITE). Madrid, 2007.

Nikolopoulo, M. (2004). Thermal confort models for urban spaces. En: Designing open spaces in the urban environment: a bioclimatic approach. Atenas: Centre for renewable energy sources.

Olgyay, V. (1988). Arquitectura y clima. Manual de diseño bioclimático para arquitectos y urbanistas. Barcelona: Gustavo Gili.

Piedecausa, B. (2013). Medida de Radón en espacio de trabajo subterráneo del Campus de la Universidad de Alicante. Informes de la Construcción. (65) 531, 301-310.

Sánchez, D. \& Sánchez- Guevara, C. \& Rubio, C. (2016). El enfoque adaptativo del confort térmico en Sevilla. Anales de Edificación, (2), 1, 38-48.
Sánchez, D. \& Rubio, C. \& Marrero, M. \& Guevara, F. \& Canivell, J. (2017). El control adaptativo en instalaciones existentes y su potencial en el context del cambio climático. Revista Hábitat sustentable, (7) 2, 617.

Serra, R. \& Coch, H. (2005). Arquitectura y energía natural. México: Alfaomega.

Spyropoulos, G. \& Balaras, C. (2011). Energy consumption and the potencial of energy saving in Hellenic office building used as bank branches - A case study. Energy and Building, (43) 4, 770-778. 